\title{
Cognitive Dysfunction in Diabetic Patients WITH SPECIAL REFERENCE TO AGE OF ONSET, DURATION AND CONTROL OF DIABETES
}

\author{
Priyam Mukherjee," Srijan Mazumdar, Soumik Goswami, Jayeeta Bhowmik, Subhro \\ Chakroborty, Sumanto Mukhopadhyay, Subhendu Jana, Amal Chakraborty, Sandip Pal, \\ Shyamal. K. Das, Jotideb Mukhopadhyay
}

IPGMEER, BINP E Calcutta University, Kolkata, West Bengal, India

\begin{abstract}
Aims of this study was to determine the relationship between diabetes and cognitive impairment is respect of the age of onset and duration of diabetes, other complication of diabetes mellitus and effect of short term glycemic control on cognitive impairment. In the clinical study 50 diabetic patients were examined clinically for evidence of cognitive dysfunction by "Kolkata Cognitive Screening Battery". The scores were compared with the normative data on global cognitive functioning in a population within an urban Indian context. Those having cognitive impairment, follow up was done for six months with adequate anti-diabetic drugs to control their blood sugar strictly (HbA1C $<7 \%)$. Those who had adequate blood sugar control were again interviewed similarly. The scores were compared with previous values. Results show that cognitive dysfunction was associated with diabetes. Recognition, fluency and immediate memory were most commonly affected. Calculation was least affected. No significant correlation found between cognitive decline with either duration or age of onset of diabetes. The cognitive decline appeared to be reversible as improvement of some mental faculties after strict blood sugar control. In conclusion we have found that cognitive decline was associated with diabetes but not directly related to the duration and age of onset of diabetes. On the other hand, control of diabetes lead to improvement of cognitive function.
\end{abstract}

Key words: Cognitive Decline; Kolkata Cognitive Screening Battery; Diabetes; Geriatric Depression Scale (GDS); HbA1C; Mini-Mental State Examination (MMSE)

\section{INTRODUCTION}

Diabetes is a systemic disease having an impact on every organ of the body including the brain with cognitive impairment being noticed in diabetic patients. Deficits have been seen in areas of psychomotor efficiency, global cognition, episodic memory, semantic memory, and working memory (Arvanitakis et al., 2004; Ryan et al., 1993). Abnormalities in cognitive functions mediated by frontal lobe (executive functions), including a number of complex behaviors such as problem solving, planning, organization, insight, reasoning, and attention, are noted in patients with diabetes (Gold et al., 1993).

${ }^{*}$ Correspondence to: Priyam Mukherjee, e-mail; drpriyam.mukherjee82@gmail.com

Author's note: Readers who are interested in other data regarding this article please write to the e-mail address above. Received April 25, 2012; accepted May 30, 2012; Act Nerv Super (Praha) 54(1-2), 67-75. 


\section{MATERIALS AND METHODS}

We have selected 50 diabetic patients attending Medicine inpatient and outpatient departments and Diabetes Clinic of SSKM Hospital (I.P.G.M.E\&R) .They all have T2DM as per ADA 2009 criteria (ADA, 2009). Those having Dementia according to DSM IV criteria, significant hearing or visual impairment and unable to participate in an interview in a meaningful manner, family history of dementing illnesses, Type 1 diabetic patient, past or current history of cerebrovascular accident or epilepsy, other risk factors known to cause cognitive impairment e.g. stage 3-5 CKD, Parkinson's Diseases, Alzheimer's disease, CLD, HIV, Hypothyroidism, Vitamin B12 deficiency and other drugs, neurosyphilis, malignancy, structural brain disease, chronic CNS infection psychiatric diseases like major depression, schizophrenia were excluded .

Patients were stratified according to age of onset and duration of diabetes. All the patients received treatment as only diet, diet and oral hypoglycemic agents (OHA), diet with OHA and insulin as per attending physician's judgment to keep $\mathrm{HbA} 1 \mathrm{C}<7 \%$.They were subjected to a structured interview in the out-patient department which included demographic information such as age, sex, literacy level, years of schooling, occupation held during their working life and mental state examination. The cognition was assessed by "KolkataCognitive Screening Battery" (Das, et al., 2006). Kolkata Cognitive Screening Battery consisted of category-based verbal fluency tests (fruits and animals), a 15 item version of the object naming test, mental state examination, calculation tests, visuo-constructional ability which included drawing the circle, diamond, overlapping rectangles, box and a set of memory tests which consisted of immediate memory, delayed and recognition of a 10 item wordlist. This test battery has already been used and validated by Ganguli and her colleagues in a rural population at Ballabgarh in north India (Ganguli et al., 1996). In addition, Geriatric depression scale (GDS) was also applied to establish the presence of hitherto undetected depression. The cognitive test battery was then administered under the direct supervision of the neuropsychologist. Geriatric Depression Scale (GDS) (Ganguli et al., 1999), which was already validated for internal consistency, was also applied. Information was corroborated with at least one close family member, usually the spouse, children, or a reliable informant closely associated with the test subject. Subsequently, all information recorded in the proforma were verified by a senior neurologist and one senior psychiatrist who checked the completeness of the information entered and in the event of any deficiency, further contact was made with the participants. The score were compared with the normative data (Das, et al, 2005) on global cognitive functioning in a population within an urban Indian context. Lowest 10th percentile score was considered as the cut-off point for identifying the significantly impaired cognitive function (naming, fluency, MMSE, calculation, memory, praxis), as described in the Kolkata Cognitive Screening Battery. After identifying the patients with cognitive impairment i.e. patients with one or more mental faculty abnormality described in cognitive screening battery were considered having cognitive dysfunction and were followed up for six months with adequate anti-diabetic drugs to strictly control their blood glucose $(\mathrm{HbA} 1 \mathrm{C}<7 \%)$. Those having adequate blood sugar control were interviewed again in the same manner with Kolkata Cognitive Screening Battery. In these patients, the scores of cognitive parameter after strict diabetes control were compared with the scores obtained prior to tight diabetes control. (Flow Chart 1). The data were collected during the period of Feb-2009 to Jul-2010.

The data of cognitive scores before and after treatment was analyzed with Mann-Whitney $\mathrm{U}$ test to analyze the mean effect of sugar control after six months. 


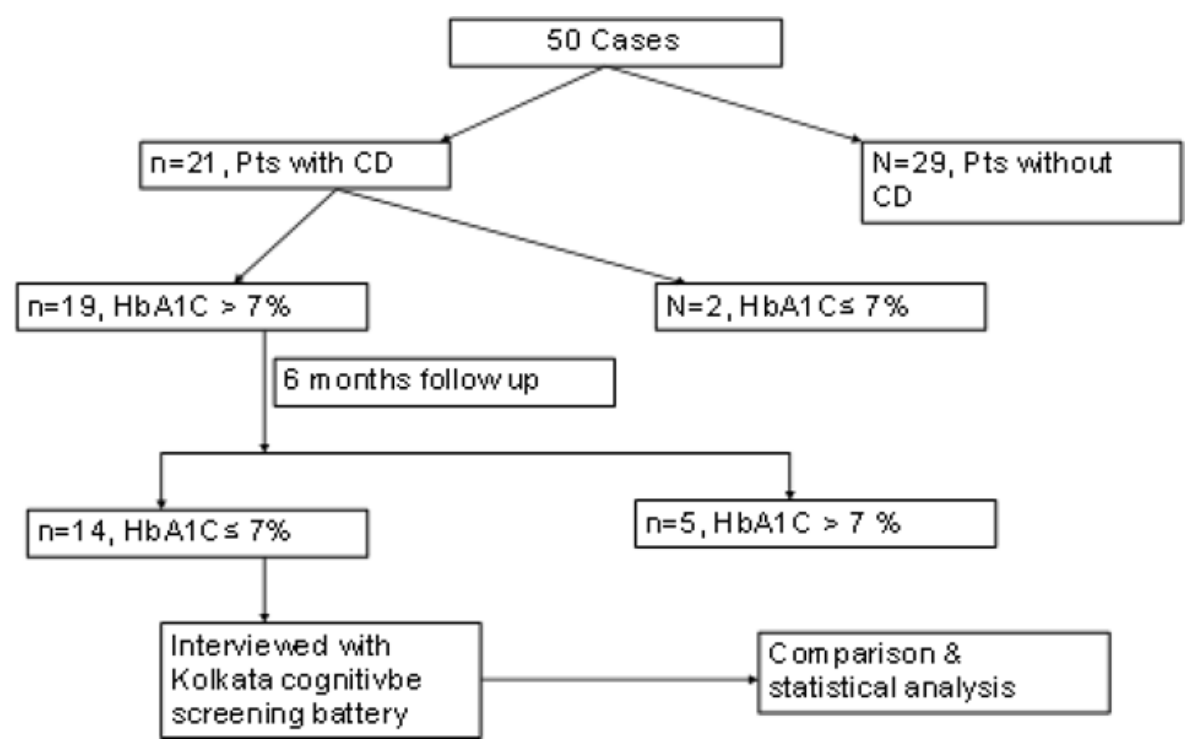

Flow Chart 1. 50 cases were followed up in the following way. Note: CD- Cognitive dysfunction.

\section{RESULTS AND ANALYSIS}

\subsection{Initial assessment}

3.1.1. 50 patients were included in study population. The mean age of study population was 58.54 years +11.6 .years $($ maximum $=82 \mathrm{yrs}$, minimum $=34 \mathrm{yrs})$. Among 50 patients $6 \%(\mathrm{n}=3)$ belonged to age group $30-39,14 \%(n=7)$ to age group $40-49,40 \%(n=20)$ to age group $50-59$, $20 \%(n=10)$ to age group $60-69,16 \%(n=8)$ to age group $70-79$ and $4 \%(n=2)$ to age group $80 \&$ above.

3.1.2. In the study group $2 \%(n=1)$ had onset of diabetes at an age of less than 30 years. $8 \%$ $(n=4)$ had onset of diabetes between $30-39$ years, $34 \%(n=17)$ each had onset of diabetes between the ages of 40-49 years and 50-59 years respectively, $14 \%(n=7)$ had onset of diabetes between $69-69$ years and $8 \%(n=4)$ patients had diabetes onset between $70-79$ years of age. Overall, most of the patients had onset of diabetes between 40 to 60 years.

3.1.3. Among 50 patients $30 \%(n=15)$ had duration of diabetes of less than 5 years, $40 \%($ $\mathrm{n}=20)$ had duration between 5 to 10 years and $30 \%(n=15)$ had duration of more than 10 years.

3.1.4. Regarding literacy level, in the age group of 30-39 years, $100 \%(n=3)$ patients had attained literacy level of class 6-12. In age group of $40-49$ years, $57 \%(n=4)$ patients had attained literacy level of graduation and above, 29\% $(n=8)$ of class $6-12$ and $14 \%(n=1)$ of class1-5. In age group of 50-59 years, $50 \%(n=10)$ had attained literacy level of graduation and above, $40 \%(n=8)$ of class $6-12,5 \%(n=1)$ of class $1-5$ and $5 \%(n-=1)$ were illiterate. . In age group of $60-69$ years, $30 \%(n=3)$ had attained literacy level of graduation and above, $50 \%$ $(n=5)$ of class $6-12,10 \%(n=1)$ of class1-5 and $10 \%(n-=1)$ were illiterate. In the age group of $70-79$ years, $12.5 \%(n=1)$ had attained literacy level of graduation and above, $50 \%(n=4)$ of class $6-12,25 \%(n=2)$ of class $1-5$ and $12.5 \%(n-=1)$ were illiterate and in the age group of 80 years \& above, $50 \%(n=1)$ had attained literacy level of class $6-12$ and $50 \%(n=1)$ of class1-5.

3.1.5. Frequency distribution of some other important parameters are shown below

3.1.6. Regarding smoking habit, among 50 patients, $68 \%(n=34)$ were nonsmoker and $32 \%$ $(n=16)$ were current smoker. 
3.1.7. Among 21 patients who had cognitive dysfunction most patients had problem in recognition ( $\mathrm{n}=17,82 \%)$ with the next most common abnormality being fluency (Fluency of fruit naming $n=16,79 \%$ \& fluency of animal naming $n=15,72 \%$ ). Immediate memory was abnormal in 13 patients $(62.5 \%)$ and later recalling was abnormal in 8 patients $(38.5 \%)$. Naming and praxis was abnormal in 9 and 4 patients respectively (43\% \& 19\%), MMSE was abnormal in 5 patients $(24 \%)$ and abnormality in calculation was detected in 2 patients only (9.5\%). (Fig1)

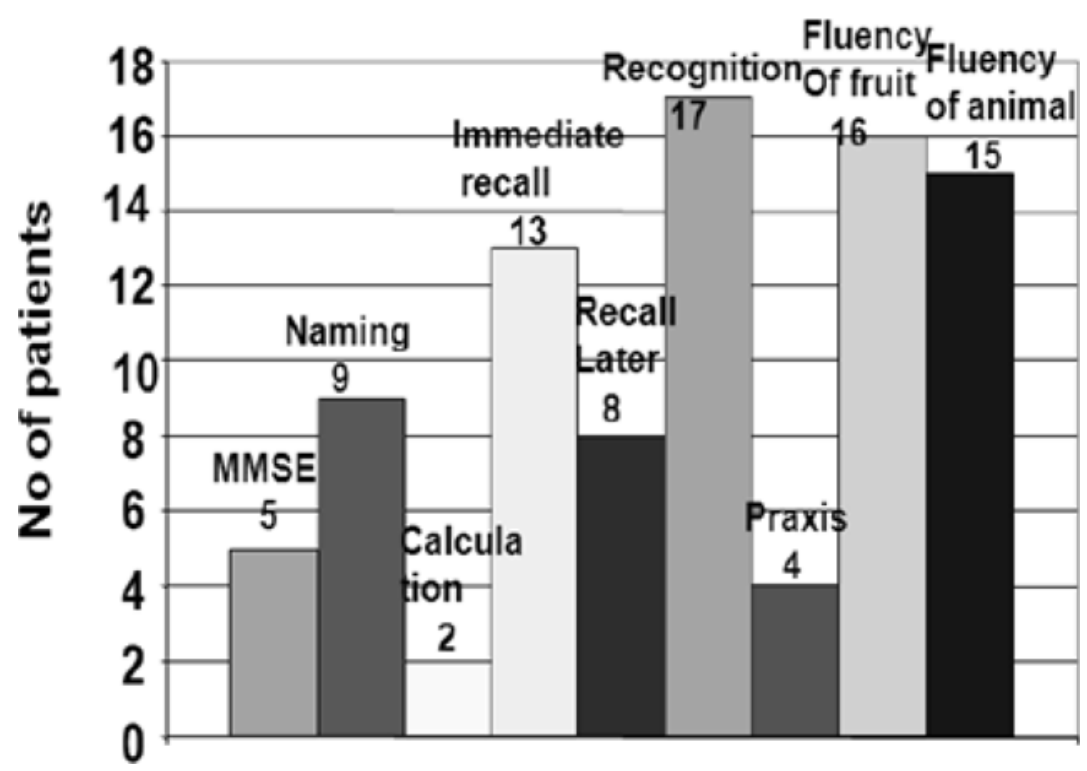

Figure 1. Impaired mental faculty.

3.1.8. Our study showed that cognitive dysfunction has positive significant correlation $(p<0.05)$ with smoking habit, nephropathy $(\mathrm{p}<0.05)$, retinopathy $(\mathrm{p}<0.05), \mathrm{HbA1C}$ (more than $7 \%)(p<0.01)$ and is inversely related to the regularity of diabetes treatment $(\mathrm{p}<0.01)$. (Table 2$)$ 3.1.9. Our study did not show any significant positive correlation $(p>0.05)$ between duration of diabetes and its age of onset with cognitive dysfunction. (Table 1)

3.1.10. There was no significant positive correlation $(p>0.05)$ between sex of patients and cognitive dysfunction in our study. (Table 1)

3.1.11. Our study did not show any significant positive correlation $(p>0.05)$ between dyslipidemia, BMI and hypertension with cognitive dysfunction. (Table 1)

\subsection{Evaluation after six months}

After the initial cognitive assessment, we tried to control the blood sugar strictly (HBA1C< $7 \%$ ) in the 21 patients having cognitive dysfunction of whom two patients had HbA1C value less than 7 while the remaining 19 patients had $\mathrm{HbA1C}$ value more than 7 . We were able to achieve an $\mathrm{HbA1C}<7$ in 14 patients out of 19 after six months. Cognitive test was again done in a similar fashion and scores were recorded and compared. The results were -

1. Improvement of mean values of Immediate recall, Fluency (Fruit), Praxis and Recognition which were statistically significant after treatment $(p<0.05)$ (Table2)

2. However, change of mean value of other parameters like MMSE, calculation, recall later, fluency(animal), fluency(total) and naming were not statistically significant after treatment $(\mathrm{p}>0.05)$ (Table2) 
Table 1. Composite table showing correlation of different variables with cognitive dysfunction.

\begin{tabular}{|c|c|c|c|}
\hline Parameter studied & Correlation with & P value & Remarks \\
\hline Age of onset of diabetes & Cognitive dysfunction & $>0.05$ & Not significant \\
\hline Duration of diabetes & Cognitive dysfunction & $>0.05$ & Not significant \\
\hline Sex of diabetic patients & Cognitive dysfunction & $>0.05$ & Not significant \\
\hline Literacy level & Cognitive dysfunction & $>0.05$ & Not significant \\
\hline Hypertension & Cognitive dysfunction & $>0.05$ & Not significant \\
\hline Dyslipidemia & Cognitive dysfunction & $>0.05$ & Not significant \\
\hline BMI & Cognitive dysfunction & $>0.05$ & Not significant \\
\hline High HbA1C $(>7 \%)$ & Cognitive dysfunction & $\leq 0.001$ & Significant \\
\hline $\begin{array}{lll}\text { Regularity of diabetic } \\
\text { treatment } & & \end{array}$ & Cognitive dysfunction & $\leq 0.001$ & Significant \\
\hline Smoking habit & Cognitive dysfunction & $<0.05$ & Significant \\
\hline Nephropathy & Cognitive dysfunction & $\leq 0.05$ & Significant \\
\hline Retinopathy & Cognitive dysfunction & $<0.05$ & Significant \\
\hline Neuropathy & Cognitive dysfunction & $>0.05$ & Not significant \\
\hline CAD & Cognitive dysfunction & $>0.05$ & Not significant \\
\hline PAD & Cognitive dysfunction & $>0.05$ & Not significant \\
\hline
\end{tabular}

Note: CAD- Coronary artery disease, PAD- Peripheral arterial disease.

\subsection{Evaluation after six months:}

After the initial cognitive assessment, we tried to control the blood sugar strictly (HBA1C $<$ $7 \%$ ) in the 21 patients having cognitive dysfunction of whom two patients had HbA1C value less than 7 while the remaining 19 patients had $\mathrm{HbA1C}$ value more than 7 . We were able to achieve an $\mathrm{HbA1C}<7$ in 14 patients out of 19 after six months. Cognitive test was again done in a similar fashion and scores were recorded and compared. The results were -

1. Improvement of mean values of Immediate recall, Fluency (Fruit), Praxis and Recognition which were statistically significant after treatment $(p<0.05)$ (Table2)

2.However, change of mean value of other parameters like MMSE, calculation, recall later, fluency(animal), fluency(total) and naming were not statistically significant after treatment ( $>0.05)$ (Table 2).

Table 2. Summary of change in cognitive parameter after 6 months.

\begin{tabular}{|c|c|c|c|c|c|c|c|c|c|c|}
\hline $\begin{array}{c}\text { Mental } \\
\text { Faculty }\end{array}$ & MMSE & Calculation & Immediate & Recall & Recognition & Praxis & Fluency & Fluency & Fluency & Naming \\
\cline { 7 - 10 } & & & recall & Later & & & (fruit) & (Animal) & (Total) & \\
\hline $\begin{array}{c}\text { Mean } \\
\text { Value } \\
\text { Before Rx }\end{array}$ & 27.5 & 3.85 & 11.5 & 2.71 & 14.5 & 7.14 & 6.71 & 6.78 & 13.86 & 13.36 \\
$\begin{array}{c}\text { Mean } \\
\text { Value After } \\
\mathbf{R x}\end{array}$ & 27.71 & 3.78 & 12.21 & 2.85 & 15.07 & 7.71 & 7.5 & 6.92 & 14.43 & 13.43 \\
\hline P Value & $>0.05$ & $>0.05$ & $<\mathbf{0 . 0 5}$ & $>0.05$ & $<\mathbf{0 . 0 5}$ & $<\mathbf{0 . 0 5}$ & $<\mathbf{0 . 0 5}$ & $>0.05$ & $>0.05$ & $>0.05$ \\
\hline Significance & $\mathrm{NO}$ & $\mathrm{NO}$ & $\underline{\text { Yes }}$ & $\mathrm{NO}$ & $\underline{\text { Yes }}$ & $\underline{\text { Yes }}$ & $\underline{\text { Yes }}$ & NO & NO & NO \\
\hline
\end{tabular}

\section{DISCUSSION}

In the study group, $42 \%(n=21)$ had cognitive dysfunction. Similar prevalence of cognitive dysfunction was reported in a previous study by Munshi et al. (2006).Their study showed that one-third of the elderly diabetic population had cognitive dysfunction as described by the CIB(clock in a box) test. In the Cardiovascular Health Study, prevalence of mild cognitive 
dysfunction was 19\% in individuals aged more than 65 years and $29 \%$ in those aged more than 85 years (Cukierman-Yaffe, et al., 2009). Cukierman et al. (2005) showed that individuals with diabetes were 1.5 times more likely to experience cognitive decline and frank dementia than individuals without diabetes. Therefore, the prevalence of cognitive dysfunction among diabetics found in our study is consistent with previous studies. The prevalence of mild cognitive impairment in the non-demented population above age of 50 years in Kolkata is 3.13 to $6.73 \%$ (Das, et al., 2005) which demonstrates greater cognitive dysfunction in diabetics compared to the non-diabetic population.

Among 21 patients who had cognitive dysfunction, most patients had problem in recognition $(n=17,82 \%)$ followed by fluency (abnormal fluency of fruit naming $n=16,79 \%$ \& abnormal fluency of animal naming $n=15,72 \%)$. Immediate memory was abnormal in 13 patients $(62.5 \%)$ while later recalling was abnormal in 8 patients $(38.5 \%)$. Naming and praxis was abnormal in 9 and 4 patients respectively (43\% \& 19\%), MMSE was abnormal in 5 patients $(24 \%)$ and abnormality in calculation was detected in 2 patients only $(9.5 \%)$.

Regarding the mental faculty abnormalities, the literature shows inconsistent results. Arvanitakis et al. (2004) and Ryan et al. (1993) showed deficit in areas of psychomotor efficiency, global cognition, episodic memory, semantic memory, and working memory which is consistent with the results of our study. Many study workers like Gold et al (1993) showed abnormalities in cognitive functions mediated by frontal lobe (executive functions), including a number of complex behaviors such as problem solving, planning, organization, insight, reasoning, and attention, in patients with diabetes. In our study we couldn't evaluate attention and executive function as there was no provision in our study tool to evaluate those functions.

Elias et al. (1997) also reported increased risk of a low score (bottom 25\%) for those with diabetes on five of eight tests (immediate and delayed logical memory, digit span forward, word fluency, and similarities) which is consistent with our study.

Arvanitakis et al. (2006) showed diabetes was not associated with abnormal episodic memory, working memory, or visuospatial ability. This result goes against the other observations. Though in our study least affected mental faculties were visuoconstructional ability and calculation.

Our study showed the presence of a significant positive correlation $(p<0.05)$ between smoking habit and cognitive dysfunction which is similar to that of previous studies. Carla Ruis et al (2009) showed smoking to be a significant risk factor for some early decrements. Cigarette smoking is known to be associated with several harmful biological processes in addition to its effects on blood vessels, such as atherosclerosis and other changes in vessel wall morphology and function (Esen et al., 2004).

We found a significant positive correlation $(p<0.05)$ between nephropathy and cognitive dysfunction. Microalbuminuria is an independent cardiovascular risk factor of particular relevance in diabetes, and there have been recent reports of inverse associations between microalbuminuria and performance on cognitive tests (De Luis et al., 2002).

In our study Pearson's correlation showed a significant positive correlation $(p<0.01)$ between HbA1C (more than 7\%) and cognitive dysfunction and also showed the presence of a significant strong positive correlation $(\mathrm{p}<0.01)$ between regularity of diabetic treatment and good cognitive performance which is consistent with results of different studies published earlier. Biessels et al. (2006) showed diabetes-related factors, such as insulin resistance and chronic hyperglycemia to be associated with impaired cognitive function.

Our study showed a significant positive correlation $(p<0.05)$ between retinopathy and cognitive dysfunction. However, there was no significant correlation $(p>0.05)$ between coronary artery disease, diabetic neuropathy and peripheral arterial disease with cognitive dysfunction in diabetic patients. An earlier study had showed that retinopathy is independently associated with poor cognitive function, suggesting that cerebral microvascular disease may contribute to the development of cognitive impairment (Wong et al., 2002). 
In contrast to previous studies, our study did not show any significant positive correlation $(p>0.05)$ between duration of diabetes and cognitive dysfunction. Ruis et al. (2009) had shown that diabetes duration was associated with the effect size (cognitive decline) of the study: the longer the known diabetes duration, the bigger the effect size which was substantiated by other studies. In the Study of Osteoporotic Fractures (Gregg et al., 2000), there was a trend of increasing risk of cognitive decline with increasing duration of diabetes, and in the Framingham study (Elias et al., 1997), each 5-year increment between diabetes diagnosis and cognitive assessment was associated with lower scores on tests of logical memory, word fluency, and similarities. However, studies have also shown cognitive outcome variable to be independent of duration of diabetes which have stirred up controversy regarding this issue (Manschot et al., 200831). In our study, the number of patients with long duration of diabetes ( $>10$ years) were much lower than patients having diabetes for less than 10 years. (15 vs. 35$)$ which may be a reason for the lack of statistical significance between duration of diabetes and cognitive dysfunction.

Pearson's correlation was unable to show any significant positive correlation $(p>0.05)$ between age of onset of diabetes and cognitive dysfunction in our study which is contrary to available evidence. Ruis et al. (2009) clearly showed that the age of diabetes onset was inversely related with performance of tasks for memory and information-processing speed in diabetic patients. Since we compared the cognitive score with age- adjusted normative data instead of taking a separate control group for comparison, we did not get any significant impact of age on cognitive function in our study. A similar explanation may also be applicable to literacy level where we did not get any significant correlation $(p>0.05)$ between lower literacy level and cognitive dysfunction though a lower cognitive score is expected with lower literacy levels.

There was no significant positive correlation $(p>0.05)$ between sex of patients and cognitive dysfunction in our study which is consistent with a previous syudy by Ruis et al (2009)

Our study did not show any significant positive correlation $(p>0.05)$ between dyslipidemia and BMI with cognitive dysfunction which was also observed in other studies.

After strict blood sugar control, memory in the form of immediate recall and recognition, fluency and visuoconstructional ability improved significantly. Grodstein et al. (2001) showed beneficial effect of sugar control on cognitive dysfunction but overall there is a paucity of data demonstrating the same.

\section{CONCLUSION}

Cognitive dysfunction is associated with diabetes with recognition, fluency and immediate memory being most commonly affected. Calculation was least affected but few patients had problem in MMSE, praxis and naming. There was strong association of cognitive decline with smoking habit, poor control of diabetes, nephropathy and retinopathy but the age of onset of diabetes and its duration did not have strong positive correlation with cognitive decline. The cognitive decline seems to be reversible as improvement of some mental faculties like immediate recall, recognition, praxis and fluency were significant after strict control of diabetes. This implies that control of diabetes may help improve cognition and help in better management of diabetes as cognitive impairment disrupts the individual's usual life style, interferes with self care activities and hampers diabetes control as well. Another implication is that older patients with diabetes may often have concomitant cognitive dysfunction resulting in suboptimal adherence to complicated diabetes treatment regimes. Our study adds another reason as to why diabetic patients should be advised to refrain from smoking. The strength of the study lies in the use of a validated cognitive scale developed from a local community based study as the popular western cognitive scales may not be appropriate in 
our local population as language, culture, education and intelligence are different from the western region.

\section{1. Limitations}

We have used 'Kolkata Cognitive Screening Battery' to evaluate cognitive dysfunction in Type2 diabetic patients attended diabetic clinic. The screening battery is able to test nine mental faculties (object naming test, mental state examination, calculation tests, visuoconstructional ability, fluency of both fruit and animal naming, and a set of memory tests which consisted of immediate memory, delayed and recognition of a 10 item wordlist.).Abnormalities in cognitive functions mediated by frontal lobe (executive functions), including a number of complex behaviors such as problem solving, planning, organization, insight, reasoning, and attention will be missed in the study group because there is no provision in the tool to pick up those abnormalities. After detailed interview we compared the cognitive scores with the normative data and we have classified patients having cognitive dysfunction when the scores were below $10^{\text {th }}$ percentile of the normative data. Now the normative data developed on an urban community based study of cognitive dysfunction among non-demented population in Kolkata. Many patients who attend our hospital are from rural population so the tool may loose some of its sensitivity \& specificity. It would be better if a rural population based data was available. The normative data what we have applied is based on patient's age, sex and literacy level. This is definitely strength of the study tool. But the normative data is proposed for population of age 50 and above. The normative data had four major groups (for age group 50-59 years, 60-69 years, 70-79 years and 80 and more years). The patients who are below age of 50 years though small in number, we have applied the normative value of most lower age group that is age group 50-59 years assuming that they may be compatible with lower age group also as age related dementia starts usually after 60 years. We have classified patients having cognitive dysfunction who had cognitive abnormality in one or more cognitive parameters in the study tool. We have not specified degree of cognitive impairment for example a patients having impaired immediate memory and a patient having impaired immediate memory, recognition and praxis are leveled similarly i.e. having cognitive impairment.Sample size was small so it may not represent the diabetic population.

\subsection{Directions for future research}

It was a hospital based study. If we can conduct a community based study with a larger number of patients it would have more robust evidence of positive effect of good glycemic control over cognition in diabetic patients.

\section{REFERENCES}

American Diabetes Association (2009). Standards of medical care in (Position Statement). Diabetes Care 32 (Suppl. 1), S13-S61.

Arvanitakis, Z., Wilson, R.S., Bienias, J.L., Evans, D.A., \& Bennett, D.A. (2004). Diabetes mellitus and risk of Alzheimer disease and decline in cognitive function. Archives of Neurology, 61, 661-666.

Arvanitakis, Z., Wilson, R.S., Li, Y., Aggarwal, N.T., Bennett, D.A. (2006). Diabetes and function in different cognitive systems in older individuals without dementia. Diabetes Care, 29,560-565.

Biessels, G.J., Staekenburg, S., Brunner, E., Brayne, C., \& Scheltens P. (2006). Risk of dementia in diabetes mellitus: a systematic review. Lancet Neurology, 5, 64-74.

Cukierman, T., Gerstein, H.C., \& Williamson, J.D. (2005). Cognitive decline and dementia in diabetes - a systematic overviewof prospective observational studies.Diabetologia, 42, 2460- 2469.

Cukierman-Yaffe, T., Gerstein, H.C., Williamson, J.D., Lazar, R.M., Lovato, L., Miller, M.E., Coker, L.H., Murray, A., Sullivan, M.D., Marcovina, S.M., Launer, L.J. et al. (2009). Relationship between baseline glycemic control and cognitive function in individuals with type 2 diabetes and other cardiovascular 
risk factors: the action to control cardiovascular risk in diabetes-memory in diabetes (ACCORDMIND) trial. Diabetes Care. 32,221-6.

Das, S.K. et al.: An Urban Community- Based Study Of Cognitive Functions Among Non-Demented Elderly Population In Kolkata: Epidemiology of Major Neurological Disorders- A Random Sample Survey in City of Kolkata: Final Report of the Project Entitled: Project period Oct, 2002- Sep, 2005 : 38- 49.

Das, S.K., Banerjee, T.K., Mukherjee, C.S., Bose. P., Biswas, A., Hazra, A., Dutt, A., Das, S., Chaudhuri, A., Raut, D.K., \& Roy, T. (2006). An urban community-based study of cognitive function among non-demented elderly population in India. Neurology Asia, 11, 47 - 48.

De Luis, D.A., Fernandez, N., Arranz, M., Aller, R., \& Izaola, O. (2002). Total homocysteine and cognitive decline in people with type 2 diabetes. Diabetes Research and Clinical Practice, 55, 185-190

Elias, K., Elias, M.E., D'Agostino, R.B., Cupples, L.A., Wilson, P.W., Silbershatz, H., \& Wolf, P.A. (1997). NIDDMand blood pressure as risk factors for poor cognitive performance: the Framingham study. Diabetes Care, 20, 1388-1395.

Esen, A.M., Barutcu, I., Acar, M., Degirmenci, B., Kaya, D., Turkmen, M., Melek, M., Onrat, E., Esen, O.B., \& Kirma, C. (2004). Effect of smoking on endothelial function and wall thickness of brachial artery. Circulation Journal, 68, 1123- 1126.

Ganguli, M., Chandra, V., Gilby, J.E., Ratcliff, G., Sharma, S.D., Pandav, R., Seaberg, E.C., \& Steven, B. (1996). Cognitive test performance in a community - based nondemented elderly sample in rural India: The Indo-U.S. Cross-National Dementia Epidemiology Study. International Psychogeriatrics, 8, 507-22.

Ganguli, M., Dube, S., Johnson, J.M., Pandav, R., Chandra, V., \& Dodge, H.H. (1999). Depressive symptoms, cognitive impairment and functional impairment in a rural elderly population in India: A Hindi version of the Geriatric Depression Scale (GDS-H). International Journal of Geriatric Psychiatry, 14, 807-20.

Gold, A.E., Deary, I.J., \& Frier, B.M. (1993). Hypoglycemia and cognitive function. Diabetes Care, 16, 958959

Gregg, E.W., Yaffe, K., Cauley, J.A., Rolka, D.B., Blackwell, T.L., Narayan, K.M., \& Cummings, S.R. (2000). Is diabetes associated with cognitive impairment and cognitive decline among older women? Archives of Internal Medicine, 160, 174-180.

Grodstein, F., Chen, J., Wilson, R.S., Manson, J.E., Nurses' Health Study (2001). Type 2 Diabetes and Cognitive Functionin Community-Dwelling Elderly Women. Diabetes Care, 24, 1060-1065.

Manschot, S.M., Biessels, G.J., Rutten, G.E.H.M., Kessels, R.P.C., Gispen, W.H., Kappelle, L.J. et al. (2008). Peripheral and central neurologic complications in type 2 diabetes mellitus: no association in individual patients. Journal of Neurological Sciences, 264, 157 - 162.

Munshi, M., Grande, L., Hayes, M., Ayres, D., Suhl, E., Capelson, R., Lin, S., Milberg, W., \& Weinger, K. (2006). Cognitive Dysfunction Is Associated With Poor Diabetes Control in Older Adults. Diabetes Care, 29, 1794- 1799.

Ruis, C., Biessels, G.J., Gorter, K.J., van den Donk, M., Kappelle, L.J., \& Rutten, G.E. (2009). Cognition in the early stage of type 2 diabetes. Diabetes Care, 32, 1261-5.

Ryan, C.M., \& Williams, T.M. (1993). Effects of insulin-dependent diabetes on learning and memory efficiency in adults. Journal of Clinical and Experimental Neuropsychology, 15, 685-700.

Wong, T.Y., Klein, R., Sharrett, A.R., Nieto, F.J., Boland, L.L., Couper, D.J., Mosley, T.H., Klein, B.E., Hubbard, L.D., Szklo, M. (2002). Retinal microvascular abnormalities and cognitive impairment in middle-aged persons: the Atherosclerosis Risk in Communities Study. Stroke, 33, 1487-92. 